\title{
De la Flexibilidad a la Cohesión Social Pasando por la Finanza*
}

\author{
Pierre Salama **
}

\begin{abstract}
The early twenty-first century differs radically from the post-war era, from 1945 to the early 1980s. Growth rates are lower in most European countries, wages are generally rising less steadily than national revenue, there is greater labour flexibility and, with a few exceptions, unemployment has levelled out on a high plateau. Financial globalisation has advanced further than its commercial counterpart, and above all is having more immediate effects. The financial markets are imposing heavy constraints on entrepreneurs to achieve high levels of appreciation, and this has serious impacts on people's working and living conditions, and ultimately on social cohesion generally. This chapter is geared both to mapping out the development of work flexibility and to an attempt to explain this phenomenon, transcending any mere analysis of the labour and employment markets. The new dominant forms in the workplace are in fact the result of several technological, social and financial constraints.
\end{abstract}

Keywords: Flexibility, Productivity, Wages, Finance, Technology.

Resumen: El período actual difiere profundamente de aquél de inicios de la posguerra y que termina a comienzos de los años ochenta. El crecimiento de la mayor parte de los países europeos es más débil, el alza de los ingresos del trabajo es en general más moderada que la del ingreso nacional, la flexibilidad se acentúa, y sin excepción, el desempleo se estanca a un nivel elevado. La globalización financiera es más compleja que la globalización comercial y sus efectos son, sobre todo, más inmediatos. Los mercados financieros imponen a los empresarios fuertes restricciones de valorización, lo cual de lejos tiene consecuencias sobre las condiciones de trabajo y de vida y, al final, sobre la cohesión social. El objetivo de este artículo es a la vez mostrar la evolución de la flexibilidad del trabajo, y de intentar comprenderla; y no se limita solamente al análisis de los mercados de trabajo y empleo. Las nuevas formas de dominación en el trabajo son, en efecto, el producto de muchas restricciones de orden tecnológico, social y financiero.

Palabras-clave: Flexibilidad, Productividad, Salarios, Finanza, Tecnología.

" CTF corresponde a Contrato a Término Fijo. CTI corresponde a Contrato a Término Indefinido.

* Economista, Profesor Universitario, Miembro del Cepn-Cnrs, Director Científico de la Revista Tiers Monde. Home Page: http://perso.wanadoo.fr/pierre.salama/. E-mail: salama@wanadoo.fr. Recebido em 19/01/06 e aceito em 31/01/06. 


\section{Introducción}

El período actual difiere profundamente de aquél de inicios de la posguerra y que termina a comienzos de los años ochenta. El crecimiento de la mayor parte de los países europeos es más débil, el alza de los ingresos del trabajo es en general más moderada que la del ingreso nacional, la flexibilidad se acentúa, y sin excepción, el desempleo se estanca a un nivel elevado. La globalización financiera es más compleja que la globalización comercial y sus efectos son, sobre todo, más inmediatos. Los mercados financieros imponen a los empresarios fuertes restricciones de valorización, lo cual de lejos tiene consecuencias sobre las condiciones de trabajo y de vida y, al final sobre la cohesión social.

El objetivo de este artículo es a la vez mostrar la evolución de la flexibilidad del trabajo, e intentar comprenderla; y no se limita solamente al análisis de los mercados de trabajo y empleo. Las nuevas formas de dominación en el trabajo son, en efecto, el producto de muchas restricciones de orden tecnológico, social y financiero.

\section{Del lado de los mercados de trabajo y empleo Recordando algunas definiciones}

El término flexibilidad reviste sentidos diferentes según los autores. La OCDE opone la "flexibilidad numérica" a la "flexibilidad funcional". La primera concierne a todas las formas cuantitativas de flexibilidad, tanto internas como externas a la empresa que tienen por objeto a los asalariados y el empleo. La segunda es de orden más cualitativo y se refiere a la adaptabilidad de la mano de obra. Estos dos tipos de flexibilidad evidencian relaciones entre ellas, pero conviene distinguir una de otra. Se puede considerar, por ejemplo, que cuando las empresas prefieren el largo plazo al corto, privilegian la retención de la mano de obra y el desarrollo del mercado primario ${ }^{1}$, es decir

\footnotetext{
${ }^{1}$ Recordemos que el mercado primario es un mercado de trabajo relativamente protegido en oposición al mercado secundario, menos protegido. Este mercado se caracteriza por una conexión relativamente débil entre el título profesional (diploma, grado) y la calificación que puede estar en el origen de una «descalificación» (déclassements, disminución de categoría laboral NT), más o menos importante, según la evoloción de la coyuntura, de la existencia de una tabla de cualificaciones, y por tanto de salarios, más o menos rígida, de un rol importante de acuerdo a la antigüedad. Una empresa grande puede hacer coexistir los dos mercados, en especial cuando ella recurre a los CTF y a las agencias que ofrecen empleos temporales. Se podría considerar, en última instancia, que los asalariados "no declarados», trabajadores «ilegales» ( wau noir») hacen parte del mercado secundario. Esta es la manifestación de una flexbilidad, sobre todo cuantitativa,
} 
interno a la empresa, lo que las conduce a buscar una flexibilidad funcional a su mano de obra ${ }^{2}$.

Aesta clasificación, Michon(1987) prefiere oponer la flexibilidad delcapital, correspondiente a una acción sobre las tareas, a la flexibilidad del trabajo. La primera se refiere a la flexibilidad cualitativa o funcional pero también a las prácticas de "leasing", deslocalización y lo que se ha llamado "terciarización" o aún "externalización", es decir "recentrar", y la venta de ciertas actividades. La segunda trata de la flexibilidad de los salarios, de la precarización de los empleos, de más libertad de despido, de la flexibilidad de tiempos de trabajo mensual e incluso anual. Se trata de dos formas complementarias de flexibilidad. "Se trata en un caso de la flexibilidad cuantitativa... En el otro de flexibilidad cualitativa, respondiendo a modificaciones de gustos y preferencias de la clientela, al desplazamiento de la demanda de un tipo de producto a otro. Los medios de estas flexibilidades no parecen semejantes: el empleo temporal, los diversos modos de flexibilidad de tiempos de trabajo y la flexibilidad de los salarios de una parte, los sistemas de fabricación flexibles de otra parte... La primera no cambia la activad de la empresa, sino mejor, flexibiliza los costos de funcionamiento, en especial los costos de la mano

máxima porque es ilícita. Remarquemos sin embargo que la existencia de trabajo «ilegal», relativamente importante en Europa del este, es poco importante en numerosos países europeos. Finalmente, cuando los trabajadores «ilegales» (no declarados) son trabajadores independientes, es difícil comparar su status respecto a los asalariados.

${ }^{2}$ Se considera con frecuencia que el debate sobre la flexibilidad en los Estados Unidos se dedica más a la flexibilidad funcional, mientras que en Europa ha estado centrado en la flexibilidad cuantitativa, en la medida en que la definición de los puestos de trabajo es menos rígida y la flexibilidad del mercado de trabajo, tanto de los asalariados como de los empleos, está menos desarrollada en Europa que en los Estados Unidos. Nótese, sin embargo, que con la adopción de las 35 horas en Francia, la flexiblidad cuantitativa se ha desarrollado a través de la renegociación de tiempos reales de trabajo en el seno de las empresas, con la eliminación de una serie de "tiempos muertos" (las pausas), de la integración de una parte de la formación en la Recuperación del Tiempo de Trabajo. La externalización creciente, por el "recentrage" sobre la materia principal, ha contribuído a una mayor flexibilidad cuantitativa (poner en competencia los trabajadores internos y los trabajadores "externos") pero también funcional (nueva organización del trabajo) no deducido mecánicamente de las restricciones tecnológicas, como se puede mostrar en numerosos trabajos sociológicos, y más raramente en los de economistas del trabajo. Sobre este punto, por ejemplo, ver el número especial de la revista Tiers Monde, dirigido por Hirata, Lautier y Salama: "La transformación del trabajo ", $\mathrm{n}^{\mathrm{a}}$ 154, 1998. Finalmente, la amenaza de deslocalización («outsourcing») de los establecimientos en los países donde a la vez el costo horario de la mano de obra es más bajo, la gestión de la fuerza de trabajo más «libre» (porque se inserta en un código de trabajo laxo), conduce a una «renegociación bajo el límite» de la flexibilidad cuantitativa (salarios y tiempos de trabajo) y cualitativa (nueva organización del trabajo) menos favorable a los trabajadores de sus establecimientos. Destaquemos finalmente que si esta deslocalización tiene lugar principalmente en las empresas utilizando empleos poco calificados, la evolución rápida de la formación de trabajadores en las economías emergentes, sobre todo asiáticas, hacia la mayor calificación en lo que a ellas respecta, en un futuro cercano, en empresas de tecnología cada vez más elevada. 
de obra. Se espera así, a través de la transferencia de los costos fijos hacia los costos variables, reducir los costos unitarios de producción, y en especial los costos de la mano de obra. La otra procura facilitar el cambio en los procesos de fabricación, reducir la inversión necesaria para el cambio, reducir los costos fijos del cambio a fin de amortiguar los procesos de fabricación más restrictivos: otra forma de reducir los costos unitarios" (MICHON, 1987, p. 10).

Esta última clasificación, que al menos es compartida por la mayoría de los economistas, parece más pertinente que la precedente. Ella abre la vía para una segunda clarificación concerniente a la plusvalía absoluta. Consiste en producir plusvalor. Sea que el tiempo de trabajo "real" sea prolongado; o, sobre todo, que la intensificación del trabajo se aumente por el aumento de las cadencias. En el primer caso, las formas más antiguas consistían en alargar la jornada de trabajo, las formas más modernas en disminuir las pérdidas en el trabajo ${ }^{3}$ (GOLLAC, 2005). En el segundo caso, la introducción de técnicas nuevas permite a la vez de disminuir el valor de los bienes gracias al aumento de la productividad del trabajo, pero también redefinir las tareas e intensificar el trabajo por unidad de tiempos, es decir a producir más plusvalor ${ }^{4}$. Se trata

\footnotetext{
${ }^{3}$ Por una reducción de los "tiempos muertos", es decir de las pausas en el trabajo, que resultan de concesiones pausas café o bien de inadecuaciones de diferentes segmentos de una línea de producción, de flujos "no tensionados", gracias a una reorganización del trabajo; en fin, calcular los tiempos del trabajo en relación al año más que la jornada.

${ }^{4}$ La intensificación del trabajo se refiere a los mecanismos de plusvalor absoluto "moderno", por oposición al plusvalor arcaico ligado al alargamiento de los tiempos de trabajo. A nivel conceptual, es distinto el plusvalor relativo, si bien los dos se traducen estadísticamente, sea directa o indirectamente, en una variación de la productividad del trabajo. El campo del primero es microeconómico y significa que en un mismo tiempo de trabajo más bienes y más valor son producidos porque la intensificación aumenta, sea por un crecimento de las cadencias, sea por una organización del trabajo que reduce los tiempos muertos, sea por una flexibilidad aumentada de la fuerza de trabajo cuando los equipos lo permiten. El campo del segundo es macroeconómico, aun si su lugar de aplicación es la empresa. En una misma jornada de trabajo, se producen más bienes gracias a la introducción de nuevos equipos. La productividad del trabajo aumenta entonces. El valor de cada uno de los bienes baja y el valor de la canasta de bienes necesarios para la reproducción de la fuerza de trabajo es reducida. Cuando uno se limita a la lectura de la evolución de la productividad del trabajo, este mecanismo de plusvalor relativo es difícil de distiguir de aquel del plusvalor absoluto, porque ambos se traducen en un mejoramiento de la productividad del trabajo. La distinción es importante para comprender el desgaste de la fuerza de trabajo: el aumento de la intensificación produce una fatiga prematura, física y cada vez más psíquica hoy día, resentida como una degradación de las condiciones de trabajo, ya que no necesariamente tiene lugar cuando el grado de tecnología aumenta. Sin embargo, sin entrar en esta discusión conceptual, en la literatura reciente se muestra que el lazo entre intensidad y productividad no es tan pertinente cuando el trabajo se puede descomponer en operacioes relativamente homogéneas: en este caso el vínculo entre producción y esfuerzo es inmediato. Cuando la organización del trabajo es más compleja, los objetivos colectivos son definidos, el vínculo entre intensidad y productivadad es más diluido porque la medida de la intensidad individual es dificil de estimar (GOLLAC, 2005)
} 
entonces de formas modernas de explotación de la fuerza de trabajo que se pueden oponer a las formas arcaicas de alargar la jornada de trabajo, porque son antiguas y limitadas. De estos dos casos de presentación se obtiene una impresión de "velocidad" creciente, un sentimiento de estrés, un aumento en el número de enfermedades nerviosas de muchos trabajadores sometidos al desgaste precoz de su fuerza y de su capacidad de trabajo.

\section{Globalización comercial y las nuevas limitaciones}

La modernización del aparato productivo ocurre con posterioridad a la apertura creciente de las economías, y a la introducción masiva de bienes de equipo sofisticado. Ella estimula modificaciones sustanciales de la organización del trabajo. Sin embargo, hay que desconfiar de un enfoque en términos de determinismo tecnológico que justificaría, en nombre de la modernización necesaria, los trastornos en la organización del trabajo. Se dice que los grados de libertad en la elección de esta organización existen: es frecuente observar que con un mismo conjunto tecnológico se tienen modos diferentes de organización del trabajo entre filiales de una misma firma multinacional, instaladas en diferentes países produciendo un bien similar.

La naturaleza de los productos fabricados incide igualmente en la organización del trabajo y en la búsqueda de una flexibilidad "funcional", o cualitativa, apuntando a una adaptabilidad mayor de la mano de obra empleada. Se dice que a partir de un cierto nivel de poder de compra, la demanda cambia: los productos diversificados tienden entonces a desplazar los productos estandarizados. Las relaciones entre la demanda y la oferta se sitúan pues en niveles inferiores. La diversificación de la demanda y el énfasis puesto sobre la calidad modifica la manera de concebir la gestión de los stocks y los plazos de entrega. La organización de la producción cambia: los flujos se vuelven más intensos y los stocks disminuyen. La organización del trabajo tiende entonces a cambiar profundamente: el trabajo prescrito es reducido, la demanda de equipo aumenta, al tiempo que la polivalencia se acrecienta. La precariedad (BARBIER, 2002, 2004), el trabajo anualizado, la intensificación y la competencia (más que la calificación) aumentan. La particularidad aquí es que la inversión crece poco con relación a los años ochenta; la reorganización del trabajo, y también los bienes de equipo tecnológico nuevos, tienen un papel más destacado en el aumento de la 
productividad, que el propio aumento de la formación bruta de capital fijo. En condiciones de competencia intensa, la reducción de los costos unitarios del trabajo, por falta de inversiones suficientes, pasa cada vez más por la búsqueda de una flexibilidad creciente de la fuerza de trabajo, sin que ello esté necesariamente ligado a la naturaleza de las tecnologías utilizadas. Y como la insuficiencia de inversiones se explica en parte por los arbitrajes en favor de las actividades financieras, el peso más elevado de la finanza en el balance de las empresas conlleva a un mayor peso en la búsqueda de una flexibilidad mayor el trabajo (ver arriba).

\section{Cuadro 1 - Cuadro sintético de cambios en la organización del trabajo a partir de una reestructuración ${ }^{5}$}

\section{Organizaciones Fordistas}

Organizaciones flexibles "ohnistas"

\section{Intensificación de la división}

del trabajo

2. Trabajo prescrito

3. Sistema organizacional rígido y centralizado

4. Autonomía personal del

trabajador cercana a cero

5. Trabajo repetitivo y monótono

6. Funciones que exigen una

calificación mínima

7. Organización del trabajo: un

puesto - un hombre- una tarea

8. Compromiso débil de los

trabajadores dentro de la empresa

9. Tasa de turn-over elevada y ausentismo

10. Altos índices de rechazo

1. Intensificación del ritmo de trabajo

2. Mayor participación de los trabajadores en la organización y control de los procesos

3. Más responsabilidad para los trabajadores

4. Células, minifábricas, polivalencia y

multifuncionalidad

5. Funciones que exigen una mayor calificación

6. Organización del trabajo sobre la base de equipos

7. Compromiso necesario de los trabajadores con la empresa.

8. Reducción de la tasa de turn-over y de ausentismo

9. Reducción sustancial de los índices de rechazo

10. Reducción de conflictos entre empleadores y trabajadores

11. Conflictos frecuentes entre

empleadores y trabajadores

\footnotetext{
${ }^{5}$ Es evidente, pero mejor vale recordar, que estas formas de organización del trabajo conciernen a las grandes y medianas empresas y de hecho excluyen a las pequeñas. Una gran parte del mundo del trabajo no concierne al "ohnismo" y sufre las formas de flexibilidad menos sofisticadas, como una gran precariedad, y por tanto la fuerza de trabajo es poco protegida, al estar muy poco organizada. Añadiendo finalmente lo relativo a las grandes y medianas empresas, una tal presentación presenta al "ohnismo" de manera un poco idílica en lo que concierte a los puntos 7 y 10. Nótese con Azkenazy (2005): "...la rotación de puestos de trabajo no permite a los asalariados de la industria y de los servicios asimilar las reglas de seguridad o ergonomía, implícitas o explícitas, y establer las estrategias personales de preservación, propias a cada puesto" 0 aún "...varios indicadores hacen pensar sobre disfuncionamientos asociados a prácticas innovadoras: de tensiones con los colegas, la jeraquía o los clientes, aislamiento, o recibir órdenes contradictorias.”
} 


\section{Una pregunta: la inestabilidad, un problema: los contratos a término fijo}

Como acabamos de ver, la flexibilidad reviste varios sentidos que pueden prestarse a confusión. También, a fin de ser precisos, centraremos el análisis particularmente en dos puntos: ¡la tendencia hacia una flexibilidad mayor acrecienta la inestabilidad en un período largo? ¿Cómo se articulan los movimientos entre las diferentes formas de contrato de trabajo (a término fijo y a término indefinido) y el desempleo?

1. Se podría pensar a priori que el período reciente se caracteriza por una mayor inestabilidad del empleo en Francia. Numerosos estudios lo muestran, especialmente el de Germe (2003). Un análisis reciente (L'HORTY, 2004) discute este análisis. Porque se trata de una paradoja, y dado que la argumentación nos parece sólida, privilegiaremos este estudio y presentaremos aquí sus principales resultados.

La inestabilidad ${ }^{6}$ no se acentúa en un largo período (1969-2002) de manera estructural, tanto por los empleos públicos y sobretodo por los empleos privados. Sobre este período se puede distinguir dos fases: una, que va hasta 1993, donde el riesgo de perder el empleo crece; y la otra, desde 1993, donde este riesgo disminuye. En el seno de estas tendencias el riesgo de perder el empleo varía en sentido inverso a la coyuntura; cuando ésta mejora, el riesgo disminuye, cuando la recesión aparece el riesgo aumenta. Los resultados obtenidos son similares, a pesar de que se limitan al campo de análisis de los empleos salariales privados sobre un período más corto (19822002). Estabilidad estructural, fluctuación según la coyuntura.

\footnotetext{
${ }^{6}$ Medida a través de las personas sin empleo, entre aquellas que ocuparon un puesto el año anterior, utilizando las preguntas retrospectivas y la encuesta en panel.
} 


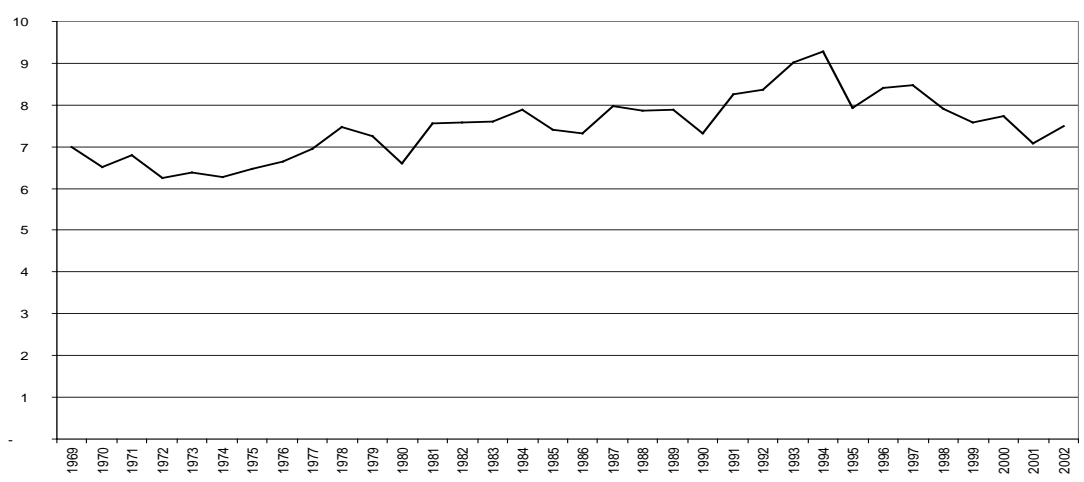

Gráfico 1 - Riesgo de perder el empleo (1969-2002)

Campo: empleo total.

Método: preguntas retrospectivas

(situación en el mes de marzo del ańo anterior al de la encuesta).

Interpretación: 7,5\% de los activos ocupados en el 2001 han dejado su empleo en el 2002.

FUENTE: Encuesta de empleo, Insee, LHORTY, 2004, p. 5.

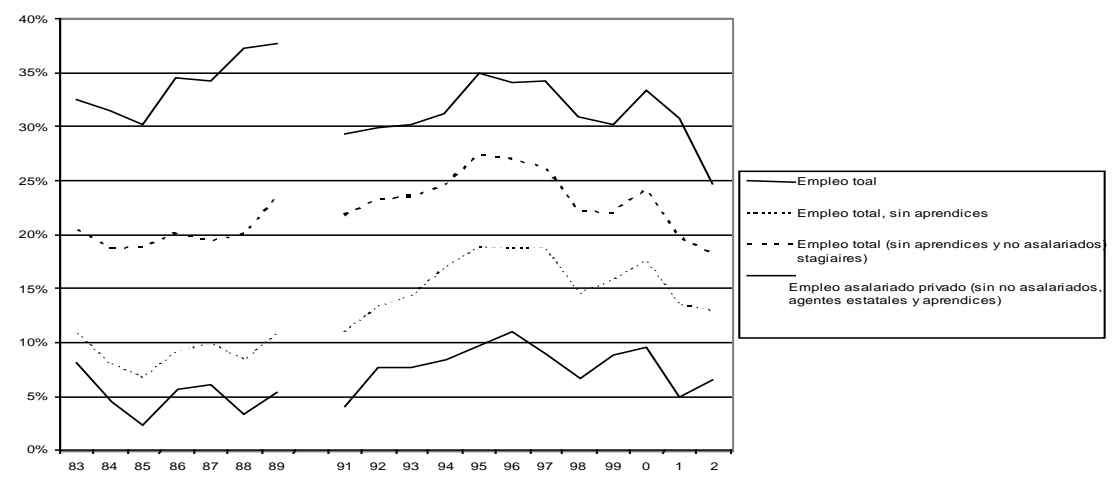

Gráfico 2 - Período relativo de inestabilidad (1982-2002) según los dos enfoques Campo: arriba, leyenda al lado del gráfico.

Método: preguntas retrospectivas y datos de la encuesta en panel.

Interpretación: para los asalariados del sector privado, el riesgo de perder el empleo medido por la cuenta de empleo en panel es $25 \%$ más bajo que aquél medido con la pregunta retrospectiva en 2002.

FUENTE: Encuesta de empleo, Insee, L'HORTY, 2004, p. 8

Esta conclusión es, sin embargo, muy global: es conveniente ser un poco menos "macro" y analizar los movimientos en el seno de diferentes categorías. 
Dicho de otra forma, estos resultados no contradicen una heterogeneidad posible frente al riesgo de diferentes categorías. Una primera observación: el riesgo de perder el empleo varía según la edad: alto para los que tienen menos de treinta años y más de cincuenta, si bien en baja para estos últimos (otro resultado inesperado...), menos alto para los asalariados que se sitúan en el tramo de edad entre los treinta y los cincuenta años. La antigüedad interviene igualmente: los contratados por último son los más vulnerables ${ }^{7}$. Como se podría aquí esperar, el riesgo está correlacionado a la calificación ${ }^{8}$. Lo que aquí es más original es el período observado en el riesgo de perder el empleo, del orden del $50 \%$ entre trabajadores calificados y no calificados, el cual no aumenta en el período (1982-2002) y tiende a reducirse ligeramente en los años noventa. Finalmente, hay diferencias importantes según los sectores de actividad: más alto en los servicios a las personas, alto en el comercio y los servicios a las empresas, menos alto en la industria, para los asalariados del sector privado, con una tendencia ligera hacia la convergencia. Así, la antigüedad protege menos que antes y el riesgo de perder el empleo aumenta en los veinte últimos años, al contrario de la edad avanzada (más de cincuenta años). Pero la calificación sigue protegiendo a los trabajadores de una movilidad forzada y el riesgo de perder su empleo no se acentúa sobre un período largo; de igual manera si ella fluctúa de manera inversa con la coyuntura.

Por último se puede, evidentemente, combinar los criterios: en el sector de los servicios, pero también en el de comercio-servicios a las empresas, los trabajadores calificados pertenecientes a categorías de edad intermedia (treinta - cincuenta años), con más de cinco años de antigüedad, tienen el $2 \%$ de chance de perder su empleo de un año respecto al otro. Por el contrario, los jóvenes poco calificados, con una antigüedad inferior a cinco años de trabajo en el sector de los servicios tienen un 20\% de riesgo de perder su empleo de un ańo respecto al otro (LHORTY, 2004, p.14). La movilidad de los trabajadores es entonces muy diferente según las categorías sociales, L'Horty (2004) cita el estudio de Amossé que muestra que el 41\%

\footnotetext{
${ }^{7}$ En el 2002, el riesgo de perder su empleo es, para los trabajadores que tenían menos de cinco años de antigüedad, el doble del correspondiente a los trabajadores más antiguos.

${ }^{8}$ Veremos sin embargo, que en el caso del Reino Unido, las personas que tenían poca calificación son proporcionalmente menos importantes que el conjunto de personas para los empleos temporales, que no es el caso en Francia y España. Esta paradoja se explica en parte por la menor protección a los trabajadores que tiene un CTI (ORTEGA, 2004, p.11)
} 
de los ejecutivos trabajan dentro de la misma empresa desde el comienzo de su carrera, contra el 5\% para los empleados no calificados. Buen ejemplo, si se trata de dar uno, de la existencia de dos mundos separados, el mercado primario y el mercado secundario'.

De manera general, los trabajadores que sufren más el riesgo de perder su empleo se benefician en este período de una reducción de tal riesgo, al contrario de aquellos que estaban más protegidos y experimentan un riesgo creciente. La paradoja es que el sentimiento de exclusión aumenta; prueba que este sentimiento no es alimentado solamente por la movilidad impuesta al trabajo, sino también por las condiciones del trabajo, el status de ese trabajo, del interés de ese trabajo ligado al nivel de remuneración, la pobreza estando cada vez más ligada al trabajo.

2. Analizando el caso de Francia, Goux (2000) muestra que los Contratos a Término Fijo (CTF) y los Contratos a Término Indefinido (CTI) no son sustituibles, pero sí complementarios. Goux establece cuatro observaciones: 1. las empresas deben adaptar el volumen de sus empleos según las evoluciones de coyuntura y sus resultados, estos dos factores no están necesariamente ligados. 2. Lo esencial de estos ajustes cae sobre los asalariados de los cuales la antigüedad tiene un coeficiente menor a un año. Así, los asalariados que tienen menos de un año de antigüedad tienen un coeficiente de variación ${ }^{\mathbf{1 0}}$

\footnotetext{
${ }^{9}$ Para ser un poco más completo, habría que analizar la "descalificación". Este es definido por la diferencia que puede existir entre los títulos (diplomas, grados) y la calificación real, tal como es definida en las tablas de clasificación de las ramas y las empresas. Cuando la recesión llega, el "shock macroeconómico" que sufren las empresas las conduce a ajustar el volumen de empleo. El ajuste se efectúa más fácilmente por las cantidades que por el precio (salarios), las empresas van a aplazar contrataciones. Las colas son una oportunidad para operar la descalificación: los más calificados serán contratados si aceptan ser descalificados, los menos calificados serán relegados al fin de la lista, al final de la cola. De esta forma, la productivad del trabajo podrá crecer gracias al reclutamiento de graduados (diplomados), y el costo unitario del trabajo bajar. De 1990 a 2000, se observa que los más afectados por esta descalificación en Francia son los jóvenes de 18 a 29 años; por orden decreciente, se trata de graduados de bachillerato técnico, bachillerato general y títulos de técnicos, de segundo ciclo. Los menos afectados son quienes se sitúan en los dos extremos: graduados de tercer ciclo o de las escuelas de ingeniería; de un lado, los de títulos de capacitación técnica, del otro (ver Gautié; Nauze-Fichet, 2000). Finalmente, la propensión a la descalificación depende también del mercado al cual accede el trabajador buscando empleo. Es por ejemplo más difícil dejar el mundo de "pequeños trabajitos", precarios por escencia, que llevan a descalificación pronunciada, para a un mercado primario más estable, donde las perspectivas de carrera pueden limitarse fuertemente debido a los efectos de la descalificación de origen. Para concluir sobre este punto, para un desempleado el desclasamiento es con frecuenda el precio a pagar por encontrar un empleo. Ello puede ser talvez muy elvado y traducirse en una baja de los salarios reales, caundo el sistema de protección de los trabajadroes es débil y la movilidad fuertemente restringida, como lo muestra el ejemplo de los países anglosajones (ver arriba).
}

${ }^{10}$ Determinado por la relación entre la desviación mediana, multiplicada por 100. 
de $56.6 \%$ contra 8.1 para los otros. Los asalariados que tienen menos de un año de antigüedad representan el $10.9 \%$ de la población asalariada, pero su volumen varía más de $50 \%$ en promedio de un mes a otro. 3. Variable de ajuste, los CTF son igualmente una vía de pasaje hacia los empleos más estables del tipo CTI. 4. Finalmente, numerosos despidos de asalariados en CTF se explica por la no renovación de su contrato que, desde el punto de vista de la empresa, es menos costoso que un despido si éste hubiera tenido lugar en CTI.

Tales resultados deben ser matizados. El estudio comparado de la estructura de los empleos, según sean a término fijo o indefinidos, también según diferentes países (Reino Unido, España, Francia, ver Ortega, 2004), es rico en enseñanzas. Si se sigue a Goux (2000), deberíamos obtener resultados comparables en España y en el Reino Unido con los observados en Francia, en la medida en que estos países, siendo diferentes, no lo son lo suficiente para justificar tales diferencias: su nivel de desarrollo es comparable, las limitaciones internacionales que padecen son del mismo orden. Ahora bien, los resultaos son diferentes. El CTF no es entonces esencialmente complementario al CTI, como Goux afirma. Es un poco más que ésto. Es también la expresión de estrategias empresariales de búsqueda de la flexibilidad de los diferentes países $^{11}$.

La proporción de asalariados que tienen un empleo temporal de tipo CTF es más débil en el Reino Unido (6.7\%) que en Francia (14.5\%) y sobre todo que en España (32\%) en el 2000. Se podría esperar un resultado diferente en la medida en que las políticas liberales tienen más peso en el Reino Unido que en Francia ${ }^{12}$. La diferencia es aún más importante si se trata de trabajadores poco calificados (5.3\% contra $16.3 \%$ y $36.6 \%$ respectivamente) según Ortega (2004, p. 11).

\footnotetext{
${ }^{11}$ Es porque es un poco "corto" afirmar: "Gravar los CTF, no sería gravar un stock de empleo particular y favorecer los otros, sino gravar las capacidades de ajuste de corto plazo de las empresas" (p. 309), en la medida en que las capacidades de ajuste varían de país a país según las legislaciones y las prácticas que recaen sobre la flexibilidad.

${ }^{12} \mathrm{La}$ Ocde calcula un índice compuesto de la protección del empleo. Dicho índice incluye una serie de variables, entre las cuales están la duración del preaviso de despido injusto y las indemnizaciones correspondinetes en este caso, la duración del procedimiento de retiro y las posibilidades de reintegro. Este índice es 0,8 y 0,3 para los CTI y los CTF al final de 1986 en Gran Bretaña. Era muhco más elvado en el caso de Francia (2,3 y 3,6) y de España (2,6 y 3,5). Nótese, sin embargo, que la protección débil para los trabajadores que se tienen de un CTI, explica en parte el costo menos elevado, en términos relativos, de un emplo en CTI más bién que en CTF en Gran Bretaña y constituye así un elemento explicativo de la importancia relativa de los CTI en este país con relación a Francia y España. Nótese finalmente que el indicacor compuesto de protección del empleo está compuesto por indicadores relacionados exclusivamente a la flexibilidad numérica, sin ninguna referencia a las condiciones de trabajo ligadas a la organización del trabajo y entonces al stress que puede estar asociado, como ya lo vimos.
} 
Cuando se compara la situación de los trabajadores residentes en Francia en 1996 y 1998, se observa que el 41.2\% de los CTF se mantienen CTF dos años más tarde, el $37.9 \%$ transforma su contrato en CTI y poco más del $20 \%$ se encuentra sin empleo. La diferencia con los trabajadores que CTI: el 94.7\% se mantienen en CTI dos años más tarde, el 1,4\% conocen un deterioro de su contrato de trabajo y pasan a CTF, el 3.9\% conocen el desempleo. Para los desempleados, más de la mitad permanece en desempleo (ver figura 1, Ortega, 2004, p. 12).

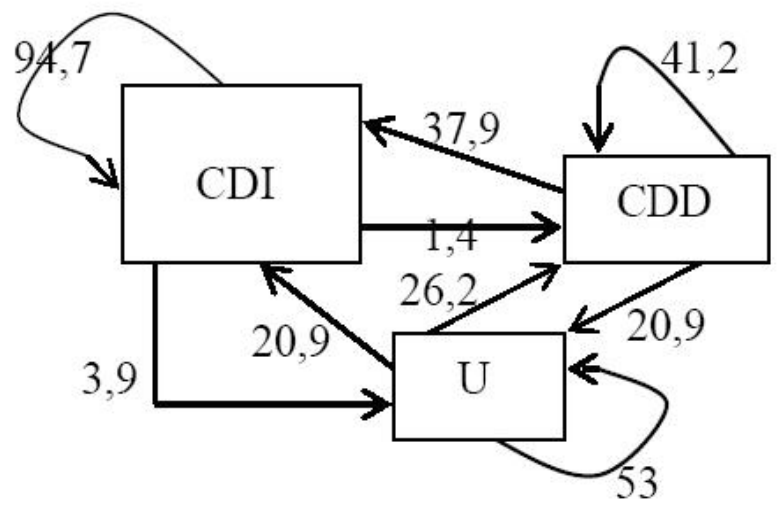

Figura 1 - Probabilidades de transición de los trabajadores en el mercado de trabajo francés (en porcentajes). Situación de los individuos en 1998 en función de su situación inicial en 1996

FUENTE: OCDE, 2002, p. 163, según el Panel Européen des Ménages

Las relaciones entre los tres polos son diferentes en el Reino Unido y en España. Los trabajadores residentes en las islas británicas tienen una probabilidad mucho más elevada que los trabajadores residentes en Francia o en España de encontrar directamente un CTI, pero su probabilidad de caer en el desempleo es igualmente más alta, o bien de obtener enseguida un CTF (ver figura 2, ORTEGA, 2004, p. 13). 


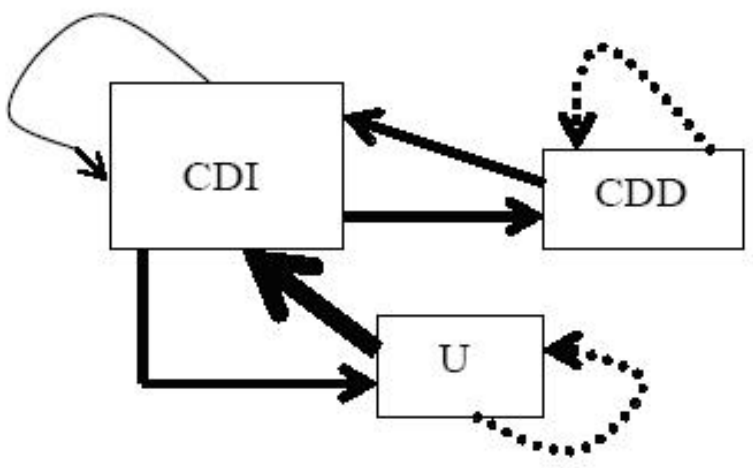

Figura 2 - Probabilidades de transición de los trabajadores en el mercado de trabajo británico comparadas a las probabilidades del mercado de trabajo francés. Situación de los individuos en 1998 en función de su situación inicial en 1996

FUENTE: OCDE, 2002, p. 163, según el Panel Européen des Menages

Les flèches à trait très épais continu (discontinu) indiquent des probabilités de transition supérieures (inférieures) de $100 \%$ à celles observées dans le cas français. Les flèches à trait épais continu (discontinu) indiquent des probabilités de transition supérieures (inférieures) de $25 \%$ à celles du cas français

Esta probabilidad más elevada alimenta un fuerte sentimiento de inseguridad en el Reino Unido, a pesar de una tasa de desempleo bajo, comparado con el que hay en España y Francia, en razón de una caída de los salarios después de un período de desempleo, cuando encuentran un nuevo empleo en CTF o en CTI. Al contrario, la probabilidad para un trabajador de conservar el empleo dos años más tarde teniendo un CTF, es más débil que en Francia.

Los trabajadores residentes en España que poseen un contrato CTI tienen una probabilidad mayor de conocer un deterioro de su situación, que los trabajadores residentes en Francia, pero una menor probabilidad de encontrarse en desempleo aún para los de CTF (ver figura 3, ORTEGA, 2004, p. 13). 


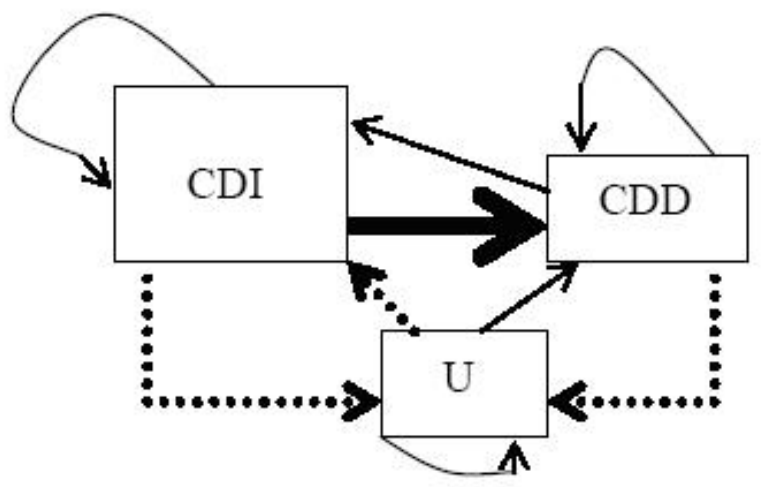

Figura 3 - Probabilidades de transición de los trabajadores en el mercado de trabajo español, comparadas a las probabilidades del mercado de trabajo francés. Situación de los individuos en 1998, en función de su situación inicial en 1996 FUENTE: OCDE, 2002, p. 163, según el Panel Européen des Ménages

Cuando se compara un período más largo (1990-2000) ${ }^{13}$, en los tres países se observa que las tasas de crecimiento promedio del PIB no son fundamentalmente diferentes $(2 \%, 1.7 \%$ y 2.6 para Reino Unido, Francia y España respectivamente); la situación de Francia es cercana a la del Reino Unido en cuanto a la creación neta de empleo (1.7\% para el Reino Unido y $1.6 \%$ para Francia), pero muy diferente a la de España (5.6\%). Pero en el caso del Reino Unido (y de España), la tasa neta es el resultado de una creación bruta y de una destrucción más importante que en Francia. La movilidad -suma de creaciones brutas y de destrucciones- es entonces más importante en España y en el Reino Unido que en Francia (14.4\%, 11.6\% y $9.1 \%$ respectivamente) sobre este período, según los trabajos de Gómez y Salvador retomados por Ortega.

\footnotetext{
${ }^{13} \mathrm{Si}$ se restringe el análisis a las grandes y medianas empresas (más de 15 trabajdores en Francia y en el Reino Unido, más de 10 en España), faltan datos comparables, lo que disminuye un poco los resultados debido a la relocalización de los trabajadores (creación bruta más destrucción). Para las empresas de menos de veinte asalariados, esto es más importante en Francia que en el Reino Unido.
} 


\section{Del lado de los mercados financieros y sus relaciones con las diferentes formas de flexibilidad}

En la actualidad no se puede comprender el auge y las nuevas formas de flexibilidad del trabajo, si no se limita el análisis al nivel del mercado de trabajo y a las condiciones de empleo. Este mercado se encuentra, en efecto, interconectado a los otros. Mercado de trabajo, mercado de bienes, mercado de capital tienen una estrecha relación y están lejos de ser independientes unos de otros. El período actual se caracteriza igualmente por un predominio del mercado del capital sobre los otros. Este dicta ciertas condiciones de rentabilidad mínima requeridas sobre el mercado de bienes, y en consecuencia, la realización de estas condiciones sólo se puede dar en el mercado de trabajo donde las formas de empleo se pliegan a estas nuevas exigencias. Existe un peso más elevado de la finanza en el balance de las empresas, lo que genera consecuencias sobre las formas de flexibilidad y su nivel. Es necesario, sin embargo, desconfiar de todo determinismo en el tema. Así como sería un error establecer una relación estable y creciente en las relaciones entre la tecnología y la flexibilidad, lo mismo sucede entre la finanza, de un lado, y la flexibilidad de otro. La finanza es en efecto una variable que juega un rol cada vez más importante desde los años ochenta, pero es difícil de medir con precisión sus efectos sobre la flexibilidad. Ella no es el único factor: el peso de las organizaciones sindicales, las formas y la capacidad de combatividad, las relaciones de una manera más general con la política, juegan también un papel importante, pero en un contexto cada vez más limitado por el peso de las finanzas y sus exigencias ${ }^{14}$.

\section{La finanza comprendida como un "Jannus"}

El aumento de las actividades financieras no es, por naturaleza, parasitario. De una manera general, las empresas se desenvuelven en un ambiente macroeconómico sobre el cual, en general, tienen poca influencia. Ellas actúan, además, en un contexto de información incompleta. Hoy día, la complejidad de la producción aumenta la incertidumbre en cuanto a la

\footnotetext{
${ }^{14}$ En el estado actual del conocimiento, sería prematuro intentar una modelización orientada a medir el peso de cada una de tales variables, por una razón simple: la definición de las variables es muy compleja. Cómo medir la flexibidad bajo todas sus formas (¿cuál indicador lo compone?), ¿qué indicadores se toman para evaluar la financiarización, la evolución de las técnicas, la resistencia de los trabajadores, etc.?
} 
rentabilidad de los proyectos. La cobertura de estos nuevos riesgos conduce al desarrollo de productos financieros igualmente complejos. En razón de ello, el mercado financiero puede permitir el desarrollo de tecnologías nuevas y asegurar en consecuencia la conversión del aparato de producción hacia la fabricación de productos industriales cada vez más sofisticados, creando productos financieros adaptados al riesgo. Las exportaciones de productos complejos necesitan no solamente de la intervención de bancos y el montaje de un "paquete" financiero complejo y original, sino también la utilización de productos financieros llamados derivados, para cubrir una serie de riesgos, entre ellos el de tipo de cambio. De esta forma la complejización del mercado financiero es, en cierta medida, la consecuencia de la complejización de la producción. Esta complejización financiera toma vuelo con la liberalización financiera (eliminar la especialización, desintermediación, desregulación). Ello tiene ciertos costos, pero permite ganancias superiores. El desarrollo de la finanza, el aumento de los productos financieros sofisticados permite entonces in abstracto el desarrollo del capital, porque el ciclo del capital es posible solamente si las actividades financieras permiten al capital productivo ser valorizado. El aumento del sector industrial necesita un desarrollo más que proporcional del sector financiero en razón de la complejidad creciente de los productos, de los riesgos crecientes a los que se expone.

Existe un vuelco hacia la "financiarización" cuando el desarrollo de estas actividades obedece más al atractivo de los nuevos productos financieros por sí mismos, que al objetivo de disminuir los riesgos ligados al financiamiento del sector productivo. Pasado ese piso, el aspecto parasitario de la finanza se puede volver importante. La financiarización es el piso a partir del cual lo financiero (el predominio del lucro sobre lo productivo) se desarrolla a expensas de este último. El sector financiero parece entonces autonomizarse del sector productivo. Como Jannus, la finanza tiene dos caras: un lado virtuoso, cuando facilita la acumulación; un lado parasitario, cuando se hace en su detrimento. Estas dos caras coexisten, una desbanca a la otra y viceversa, según los períodos, el ambiente macroeconómico (distribución de ingresos, tipos de inserción en la economía mundial, relaciones con las economías desarrolladas y los mercados financieros internacionales). 
Las vías y los medios por los cuales la finanza impone sus imperativos a lo productivo son diversas y se combinan de manera diferente, según los sectores y la coyuntura:

- En caso de un trade off, la empresa invierte cada vez más en productos financieros, reduciendo relativamente su esfuerzo en la inversión en la producción.

- En caso de que, con más frecuencia, sufra repentinas restricciones de rentabilidad extremadamente elevadas por parte de sus accionistas principales -los cuales pueden ser fondos de inversiones, cuyo objetivo es de corto plazo y se reduce a obtener ganancias crecientes- y deba desembolsar a tales accionistas los dividendos correspondientes. Si la empresa no llega a aumentar sus ganancias, su cotización en la Bolsa baja y corre peligro. Si baja o aumenta su ganancia, su cotización se vuelve objeto de especulaciones que, contrariamente a lo que se podría pensar, no constituye un juego de suma cero. La limitación financiera llega a tal punto, que ciertas empresas cotizadas en la Bolsa, las lleva a tomar la decisión de salirse de dicho mercado financiero, porque los costos son mayores que los beneficios.

- En caso de que ocurra un endeudamiento masivo en el contexto de las fusiones absorciones, lo cual ha sido particularmente importante en los últimos quince años. El endeudamiento obliga a las empresas a buscar una tasa de rentabilidad elevada que responda a las nuevas exigencias de la finanza. Si la empresa no llega a obtener sus resultados, su capitalización en Bolsa baja y el radio de endeudamiento sobre capitalización se deteriora, lo que la sitúa en un "círculo vicioso" y acentúa, en consecuencia, las dificultades para lograr una progresión rápida de sus ganancias, una reducción de sus deudas, en detrimento de sus inversiones a largo plazo y de su actividad en investigación para quebrar dicho círculo. Como lo veremos, la inversión insuficiente y la obligación de la valorización se constituyen en una ecuación difícil, en la cual la solución reside en la capacidad de la empresa para reducir la progresión de los salarios, en favor de obtener una baja absoluta de la masa salarial a través del empleo, imponiendo la flexibilidad funcional a fin de obtener, a la vez, una progresión rápida de la productividad del trabajo a través de la baja de los salarios.

Cuando el lado virtuoso de la finanza se sobrepone al lado parasitario, las actividades financieras pueden ser entendidas como "indirectamente productivas”. El desarrollo de la finanza es entonces un tanto más virtuoso, 
y genera un aumento de patrimonios ficticios cuando el crecimiento de la capitalización en bolsa es importante: el aumento de estos patrimonios acrecienta la propensión de los hogares a consumir, y este hecho ofrece un campo suplementario de valorización del capital productivo, que es lo que se puede observar en los Estados Unidos durante la presidencia de Clinton. Así, estamos lejos de una interpretación unilateral, al concebir la relación finanzaindustria únicamente desde un punto de vista de la succión de ganancias industriales operada por la finanza. La tasa de inversión aumenta y con ello el endeudamiento, facilitado por el alza de los valores poseídos por las empresas, y el aumento de su capitalización bursátil ${ }^{15}$.

Cuando los activos comprados sufren una desvalorización brutal, como consecuencia de una caída de las acciones en bolsa, el endeudamiento, confrontado a esta descapitalización de activos, se convierte en un obstáculo mayor para la consecución de la actividad "normal": el servicio de la deuda se vuelve muy difícil de asumir en las nuevas condiciones. Una situación de tal naturaleza provoca la caída de las acciones de la sociedad, haciendo aún más difícil dicho servicio. Se entra entonces en un círculo vicioso autosostenido. El efecto sobre la inversión es inmediato: disminución de la inversión, venta de activos a bajo precio, despidos, aumento débil de los salarios. Es lo que se puede observar durante el primer mandato del presidente Bush en Estados Unidos, pero también en Francia y en la mayor parte de los países europeos, con las consecuencias negativas del desplome de las bolsas de valores con sus consecuencias negativas sobre la inversión, la investigación, los salarios, y la organización del trabajo más flexible, especialmente en los sectores de nuevas tecnologías, como las telecomunicaciones.

\section{Prioridad del accionista sobre los asalariados y sus consecuencias sobre la flexibilidad}

En el contexto de la volatilidad pronunciada de los mercados financieros, la financiarización de las empresas tiene tres efectos asociados. El primero se refiere a la lucha entre el capital financiero y el capital industrial; el segundo a la distribución de los ingresos; y el tercero, finalmente y sobre todo, a la flexibilidad. Veamos a continuación:

\footnotetext{
${ }^{15}$ Este endeudamiento financia ciertamente una parte del aumento de la inversión, pero ello es provocado escencialmente por la compra de activos a precios elevados durante el proceso de reagrupamiento. En ese caso, el aumento de la finanza favorece un aumento de la tasa de crecimiento.
} 
1. El aumento de la finanza en la industria se traduce en una modificación de la reparticipación de las ganancias en favor de las finanzas. El aumento de la parte de las ganancias en el valor agregado, consecuencia directa de las obligaciones más altas impuestas a la valorización del capital, alimenta sobre todo el "apetito" del capital financiero, y de manera importante en los países europeos, con frecuencia la parte de las ganancias que restan al capital industrial son insuficientes para aumentar la tasa de formación bruta de capital fijo en la industria. Se tiene entonces un triple movimiento: aumento de la parte de las ganancias financieras en el conjunto de las ganancias, aumento de las ganancias, pero con las ganancias industriales netas de lo financiero en regresión relativa. Este triple movimiento alimenta una oposición de intereses entre empresarios industriales y financieros, al punto de que ciertos grupos industriales tratan de liberarse de estas restricciones a través de una salida de la Bolsa. Esta oposición, como veremos tiene consecuencias sobre la contradicción clásica entre el mundo del capital y el del trabajo.

2. La financiarización de las empresas provoca una tendencia a la bipolarización de los ingresos, aplastando una fracción de las capas medias en beneficio de las capas altas, lo cual es muy evidente en Estados Unidos y el Reino Unido. El régimen de crecimiento tiene una dominación financiera, que se remonta a los años noventa y tiende, en efecto, a producir una modificación de la estructura de los ingresos entre el 5 y el $10 \%$ de los segmentos más ricos de la población. Estos segmentos más desahogados perciben un aumento en sus ingresos con relación al ingreso total. El $30 \%$ que sigue se caracteriza por una separación de sus ingresos: aquellos cuyo ingreso es más alto siguen la evolución del $10 \%$ superior, pero a una velocidad menos rápida que estos últimos; los otros conocen un proceso de empobrecimiento relativo. Una fracción importante de las capas medias pierde importancia y la sociedad se aproxima a la que existía a comienzos del siglo XX. Esta es la sociedad que Gatsby -héroe de la novela de Fitzgerald- frecuentaba. Como lo recuerda Krugman, estaba caracterizada por un número importante de empleos de servicio, empleados por el 5 al $10 \%$ de la población más rica. Este "come back" manifiesta una regresión social importante. La misma constituye una ruptura con la coherencia de la sociedad, en parte fundada sobre el aumento de las clases medias. Su naturaleza tiende a fragilizar la cohesión social. Antes de volver sobre este punto importante, nótese que con esta distorsión en la distribución de los ingresos, es lógico que se afecte la estructura de los gastos. Más precisamente, los gastos suntuarios de las capas altas aumentan, favoreciendo el aumento pronunciado de los precios de los 
servicios (en especial restaurantes, hotelería, bienes raíces) más rápido que el alza del índice de precio, y del ahorro en algunos casos. Éste se refugia en los fondos que no alimentan la inversión (o lo hacen poco). Para las empresas no financieras que quieren invertir, los mercados financieros, como recursos frescos para inversión, cuestan más que lo que aportan, al punto de que muchas empresas no financieras están pensando en salirse de la Bolsa. La prioridad dada, de un lado a los especuladores, y de otro a los accionistas, tiene un costo. Es lo que veremos.

3. La financiarización de las empresas tiende a desconectar la evolución de los salarios reales de la productividad del trabajo, y a precipitar las modificaciones en la organización del trabajo en el seno de las empresas. En este sentido, constituye una ruptura con los regímenes de crecimiento de la postguerra. La desconexión salario-productividad se explica por la importancia creciente de la parte de las ganancias financieras dentro de las ganancias totales de las empresas. En un período relativamente largo se puede observar una caída sensible de los salarios en el valor agregado y una volatilidad pronunciada de las inversiones, tal como se puede ver en los dos gráficos siguientes:

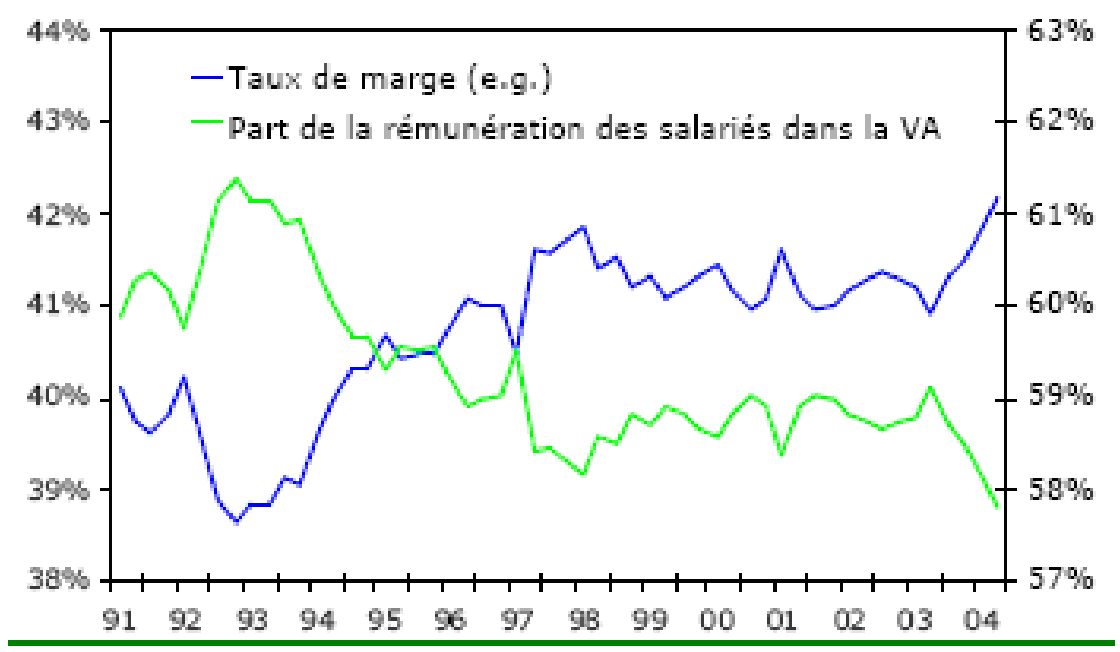

Gráfico 3 - Evolución de los salarios en el valor agregado y de la tasa de margen de beneficios en Europa

FUENTE: Eurostat. Calculs: BNP Paribas 


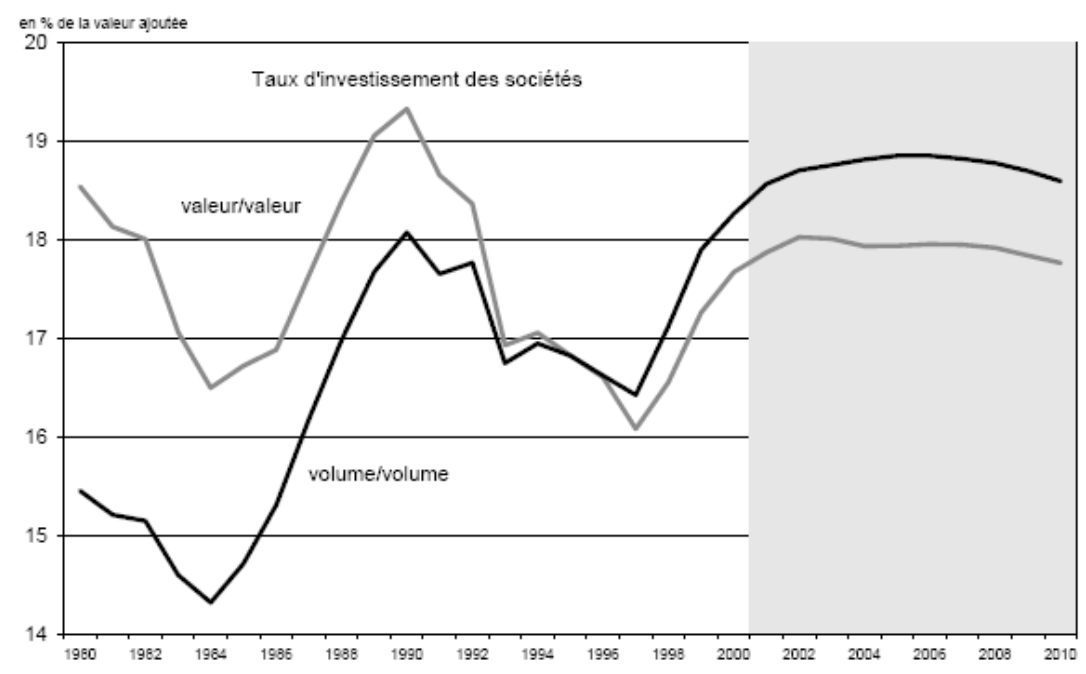

Gráfico 4 - Tasa de inversión en valor y en volumen en Francia FUENTE: OFCE 2001

El aumento de la parte de las ganancias dentro de la distribución de los ingresos, en general no ha beneficiado a la inversión. La tasa de inversión es globalmente estable, o presenta una declinación ligera sobre un período largo, aunque con una volatilidad pronunciada. De este modo el crecimiento del PIB en promedio es débil, es obtenido a partir de un crecimiento de la inversión paralela o ligeramente inferior a su participación en el Valor Agregado. De este modo, el aumento de la participación de las ganancias en el valor agregado ha servido sobre todo para financiar la deuda de las empresas y hacer desembolsar a los accionistas los dividendos mayores.

Cuadro 2 - Evolución de la estructura del valor agregado

\begin{tabular}{|cc|}
\hline $\begin{array}{c}\text { «Ganancia » } \\
\text { financiera (intereses } \\
\text { y dividendos }\end{array}$ & $\begin{array}{c}\text { Ganancia industrial } \\
\text { desembolsadss) }\end{array}$ \\
\hline
\end{tabular}


El crecimiento, aún débil, no es resultado entonces de un alza de la tasa de inversión. El alza de las ganancias en el valor agregado no constituyen, por lo tanto, "las inversiones del mañana" -para retomar una expresión ahora famosa. Ella es la precondición para que los ingresos financieros se acrecienten.

Sin embargo, las ganancias crecientes no son exclusivamente el resultado de una "moderación" salarial y de una caída de su parte en el valor agregado. El crecimiento incluso modesto y las ganancias en alza resultan de una mejora, tanto de la productividad del trabajo como de la eficacia del capital. Y ésta no se obtiene sino a partir de una flexibilización creciente, tanto numérica como funcional. No se puede entonces analizar la flexibilidad del trabajo limitándose al estudio de los mercados de trabajo y de empleo. Las nuevas modalidades de su funcionamiento se explican por una nueva lógica de valorización de conjunto, caracterizando cada vez más los regímenes de crecimiento bajo la dominación financiera. Con esta nueva lógica no es suficiente que los salarios aumenten poco, también hace falta que la flexibilidad aumente y alimente un ascenso de la productividad del trabajo, que a su vez origina un alza de las ganancias dentro del valor agregado.

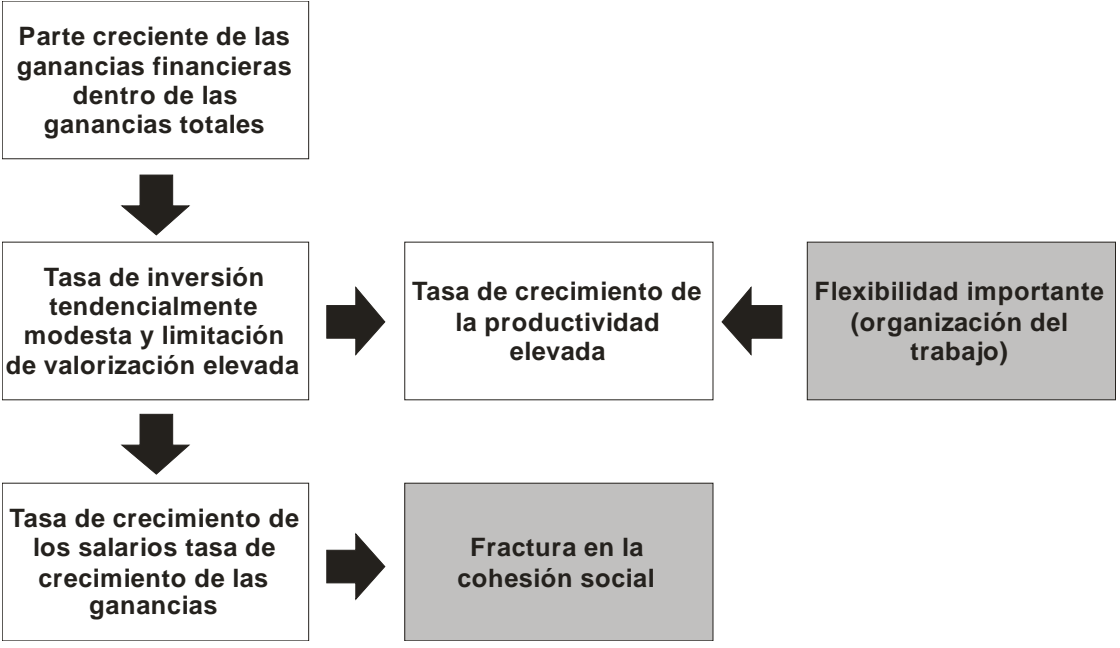

Esta alza de las ganancias dentro del valor agregado permite una "coexistencia" en el seno de las ganancias, entre las financieras y las industriales. 
Así, las ganancias industriales no pueden mantenerse o aumentar en el valor agregado, a no ser que la parte de los salarios dentro del valor agregado sea drásticamente reducida. Para comprender la lógica de este nuevo modo de crecimiento es necesario rebobinar la película. Partir de las restricciones que imponen los mercados financieros, deducir las condiciones que mantienen las ganancias industriales tanto a nivel de la caída de los salarios en el valor agregado como en la flexibilidad acrecentada del trabajo. El juego no es entonces a dos, entre "el mundo del capital y el mundo del trabajo". Es a tres, en el seno del "mundo del capital", entre el capital financiero y el capital industrial, luego con el "mundo del trabajo". En 2004 en Francia se puede observar de forma caricaturesca, con la pérdida de poder de compra, la explosión de las ganancias de un lado y la distribución de dividendos a los accionistas del otro. A tal punto que el economista jefe de la banca Ixis no dudó en escribir que "el capitalismo está a punto de autodestruirse" (Libération, 3 de marzo del 2005). Sin compartir necesariamente esta conclusión, es necesario observar que cuando la finanza tiende a dominar, la flexibilidad tiende a crecer y la cohesión social a debilitarse... en un primer tiempo. Este tiempo puede ser más o menos largo, y en términos de exclusión, de disminución del "lazo social", los efectos son considerables; pero también es posible que el crecimiento se retome, el desempleo disminuya. Estos efectos difícilmente pueden revertirse, sin embargo existen otros numerosos factores que intervienen en este "retorno" hacia una cohesión social más consecuente.

\section{Conclusión: ¿... y la cohesión social?}

Preso dentro de la presión neoliberal de los años noventa, en un contexto cada vez más internacionalizado, el Estado cumple con dificultad sus funciones (escuela, salud, justicia...) y deja con frecuencia al mercado desarrollar las desigualdades ${ }^{16}$, sin establecer de forma suficiente los contrafuegos a una exclusión social que aumenta, con la conformación de sus poblados y barrios ghettos; o la miseria moral y económica en aumento, y la desesperación creciente. La pobreza, la exclusión, no están concentradas en el no empleo; se desarrollan en el empleo, y más particularmente con los empleos

\footnotetext{
${ }^{16}$ La tendencia a la bipolarización de los ingresos puede ser "ocultada" por una estabilidad del coeficiente Gini, la curva de Lorentz siendo deformada en sus extremos.
} 
precarios. La dificultad de obtener un salario decente, la fatiga producto de la flexibilidad creciente, el desinterés frente al trabajo en contradicción con el enriquecimiento de las tareas anunciadas, acentúan la impresión de dos o tres mundos coexistentes, más allá del discurso difundido sobre la integración, la pertenencia a una unidad. La desesperanza de las capas cada vez mayores de la población puede conducirlas a buscar refugio en las salidas comunitaristas de base religiosa, ellas mismas fuentes probables de pérdida de cohesión social, de fragmentación acentuada de la sociedad y de la violencia creciente. El acceso diferenciado a los derechos es entonces percibido por los excluidos, y por aquellos cuya situación se deteriora, como la manifestación de un rechazo por parte de aquellos que aparecen como pudientes. El encerramiento parece ser una salida para una minoría, la violencia vis-a-vis de los otros pero también vis-a-vis de si mismo (autodestrucción de su entorno propio, ya pobre en servicios públicos y sociales). Una moral "flexible" se introduce entonces en una parte de la juventud excluida, seguida de una alteración de los modelos de referencia, cuyos efectos destructores sobre la cohesión social pueden ser importantes.

Frente a las manifestaciones de desasosiego social, las vías cada vez más buscadas y presentadas como las únicas eficaces son las de desarrollar la represión más que la prevención; oponer el trabajo, sea cual sea, e imponerse frente a las ventajas logradas por los subsidios a los desempleados sospechosos de holgazanería y de parasitismo. Detrás de la política represiva se desenvuelve una filosofía que le atribuye al Estado del Bienestar (welfare) la responsabilidad principal del aumento de la criminalidad. Este Estado sería en efecto permisivo, desestimulando el trabajo, el esfuerzo y "disculpando" la violencia a causa de la insuficiencia de los gastos sociales. Se trata entonces de culpabilizar al pobre ${ }^{17}$ y de imponer una mutación del "welfare" hacia el "workfare", estimulando a los pobres a trabajar más que a esperar la ayuda social. Cuando no hay una ayuda social, los pobres que no desearan trabajar serían tentados por la violencia para asegurar su supervivencia, por lo que deberían ser reprimidos, y de esta forma se pasaría de una atrofia deliberada del Estado social a una hipertrofia deliberada del Estado penal. Se observa

\footnotetext{
${ }^{17}$ Según Herrntein R., psicólogo de Harvard y autor de un libro famoso: The Bell Curve, la criminalidad es el producto de una depravación mental y moral que se encuentra sobre todo en aquellos que no se benefician de un IQ elevado, es decir, principalmente los pobres. No sirve entonces para nada para reducir las inegualidades «basadas en la naturaleza» a través de las políticas sociales.
} 
cómo este debate entre prevención y represión está lejos de ser neutro, al tiempo que detrás de estas cuestiones, que pueden aparecer como técnicas (más gasto social, más represión), existe un debate sobre la elección de sociedad.

Una sociedad sólo puede concebirse en su movimiento, en sus mutaciones. La cohesión social, porque ella es un proceso, de hecho es continuamente puesta en cuestión. Cuando este movimiento se hace de manera que puede aparecer como arbitrario e injusto por una fracción importante de la población, entonces la cohesión social sale debilitada, fracturada.

Es posible limitar estas fracturas si este movimiento es entendido, aceptado. Para que lo sea, aún hace falta que haya participación, responsabilización, no imposición. Una mayor flexibilidad no va necesariamente contra la cohesión social, si ella es negociada y no impuesta, si ella no tiene sólo por objetivo que progrese la satisfacción de los accionistas y de los financieros en detrimento del trabajo. Solamente cuando la flexibilidad es impuesta, no negociada -debido a razones fuertemente influenciadas por los intereses de la finanzalas desigualdades crecen y los ingresos del trabajo aumentan poco; cuando la finanza se dispara, ella mina el interior de la cohesión social. Y es porque hoy la flexibilidad es poco negociada, e incluso impuesta en numerosos países europeos - consecuencia en parte de la dominación de lo financiero sobre lo productivo-, que ella es considerada fuente de pérdida de cohesión social, minándola desde el interior. Pero es también porque la cohesión social sólo puede ser entendida en su movimiento, que se puede considerar que este proceso no es lineal. Las luchas que pueden generar a la vez la búsqueda de flexibilidad y las "insoportables" desigualdades reveladas, son también constitutivas de una posible cohesión social, gracias a una concientización creciente de aquellos que luchan.

La cohesión social sólo puede ser comprendida en su movimiento. Una fase de baja de la cohesión social puede ser seguida de una fase de consolidación de la cohesión bajo la condición del crecimiento; los ingresos y sobre todo el empleo aparecen de nuevo. La baja no es entonces irremediable. La reducción masiva del desempleo, el aumento del poder de compra, no tener temor de perder el empleo y las perspectivas de carrera mejoradas, todo eso puede ocurrir después de un ataque severo contra los derechos adquiridos del trabajo. La flexibilidad impuesta, fuente de la pérdida de cohesión social, se transforma entonces en su contrario y deviene en una flexibilidad aceptada 
en razón de los beneficios obtenidos en términos de la mejora del nivel de vida. Este viraje constituye con frecuencia un argumento para legitimar más la flexibilidad en los países donde todavía no se han alcanzado los niveles comparables a aquellos obtenidos en los países que tienen hoy día un quasi pleno empleo, un alza de los salarios reales... Y es aquí donde favorecer más la flexibilidad será una opción, con el riesgo de debilitar en gran medida la cohesión social; pero a largo plazo puede ser económicamente eficaz. ¿Se debe aceptar el sacrificio de una generación de trabajadores sometidos en un primer tiempo a empleos cada vez más precarios, mal pagos, indecentes, o se debe preferir aceptar jugar la carta de la negociación a fin de aceptar una flexibilidad necesaria, a riesgo de perder en eficacia en lo inmediato, pero en función de beneficios para preservar la cohesión social? 


\section{Referencias Bibliográficas}

ASKENAZY, Philippe. Partage de la valeur ajoutée en rentabilité du capital en France et aux Etats-Unis: une réévaluation. Economie et Statistique, INSEE, n. 363-364-365, 2003.

. Sur les sources de l'intensification. Revue Économique, 2005.

BARBIER, Jean Claude. A survey of the use of the term précarite in french economics and sociology. Document de Travail, n. 19. Disponible dans: http://www. cee-recherche.fr. 2002

. A compative analysis of "employment precariorness" in Europe. Document de Travail, 2004. mimeo.

. Citenzenship, flexibility and the diversity of approaches to activing social protection in Europe. Document de Travail, Conseil de l'Europe, 2004, mimeo.

BNP PARIBAS. Allemagne: vers une flexibilité accrue du marché du travail. Nov. 2004.

GAUTIÉ, Jérôme; NAUZE-FICHET, Emmanuelle. Déclassement sur le marché du travail et retour au plein emploi, dans plein emploi. Conseil d'Analyse Économique, n. 30, 2000.

GERME, J-F. (Dir). Les mobilités professionnelles: de l'instabilité dans l'emploi à la gestion des trajectoires rapport de l'atelier "mobilités professionnelles" du groupe "prospective des métiers et qualifications". La Documentation Française, 126 p., février. 2003.

GOLLAC, Michel. L'intensité du travail, formes et effets. Revue Économique, 2005.

GOUX, Dominique. La place des CDD et des CDi dans le fonctionnement du marché du travail in plein emploi. Conseil D’analyse Économique, n. 30, 2000 .

HIRATA, Helena; LAUTIER, Bruno; SALAMA, Pierre. (Sous la direction): les transformations du travail. Revue Tiers Monde, Ed. PUF, n. 154, 1998. 
L'HORTY, Yannick. Instabilité de l'emploi: quelles ruptures en tendance? Document de travail, Les papiers du CERC, n. 1, 2004.

MICHON, François. Temps et flexibilité, le temps de travail dans le débat sur la flexibilité. Cahiers économiques de Bruxelles, 1987.

OFCE. Pressions dominées: perspectives 2004-2005 pour l'économie mondiale. Revue de l'OFCE, n. 91, 2005.

ORTEGA, Javier. Le marché du travail français dans (ou à travers) le mirage britannique et espagnol. Les papiers $d u$ CERC, Document de Travail, n. 2, 2004.

PLIHON, Dominique. L'émergence du capitalisme actionnarial. Ecoflash, n. 154, 2000. 